\title{
Rough values of Piatetski-Shapiro sequences
}

\author{
Yıldırım Akbal ${ }^{1}$
}

Received: 11 August 2016 / Accepted: 4 October 2016 / Published online: 11 October 2016

(C) Springer-Verlag Wien 2016

\begin{abstract}
An integer is called $y$-rough if it is composed solely of primes $>y$. Let $L$. be the floor function. In this paper, we exhibit an asymptotic formula for the counting function of integers $n \leqslant x$ such that $\left\lfloor n^{c}\right\rfloor$ is $y$-rough uniformly for a range of $y$ that depends on $1<c<2229 / 1949$.
\end{abstract}

Keywords Piatetski-Shapiro sequences · Rough numbers · Exponential sums

Mathematics Subject Classification Primary 11N25; Secondary 11L07

\section{Introduction}

Piatetski-Shapiro was the first to show that the primes in the sequences of the form $\left\{\left\lfloor n^{c}\right\rfloor\right\}_{n \in \mathbb{N}}$ obeys an asymptotic law whenever $1<c<12 / 11$ (see [8]), hence the name. Since then, the admissible range of such values of $c$ has been extended by many authors, and currently the best known result is $1<c<2817 / 2426$ due to Rivat and Sargos [9] (see also [10])

In this paper, our motivation is to study a slightly general problem: the distribution of rough values of Piatetski-Shapiro Sequences. We advertise this problem as a generalization of counting primes in Piatetski-Shapiro sequences, because, there is a

Communicated by J. Schoißengeier.

During the preparation of the paper the author was supported by TÜBİTAK Research Grant No. $114 \mathrm{~F} 404$.

$\triangle$ Yildirım Akbal

yildirim.akbal@bilkent.edu.tr

1 Department of Mathematics, Bilkent University, Bilkent, 06800 Ankara, Turkey 
trivial one-to-one correspondence between integers $1<\left\lfloor n^{c}\right\rfloor \leqslant x$ such that $\left\lfloor n^{c}\right\rfloor$ is $x^{1 / 2}$-rough and those primes of the form $\left\lfloor n^{c}\right\rfloor$ lying in the interval $\left(x^{1 / 2}, x\right]$.

To state the theorem, some notation is in order.

For $c>1$ non-integral and for $\left\lfloor x^{c}\right\rfloor>y \geqslant 2$ real numbers, we define

$$
\Phi_{c}(x, y)=\#\left\{n \leqslant x: P^{-}\left(\left\lfloor n^{c}\right\rfloor\right)>y\right\},
$$

where $P^{-}(n)$ denotes the least prime divisor of $n$ with the convention that $P^{-}(1)=\infty$. We let

$$
\Psi(x, y)=\#\left\{n \leqslant x: P^{+}(n) \leqslant y\right\},
$$

where $P^{+}(n)$ denotes the largest prime divisor of $n$ with the convention that $P^{+}(1)=$ 0 . Given $\varepsilon>0$, we set

$$
H_{\varepsilon, c}=\left\{(x, y): x>x_{0}(\varepsilon, c), \quad \exp \left\{\left(\log \log x^{c}\right)^{5 / 3+\varepsilon}\right\} \leqslant y \leqslant x^{c}\right\} .
$$

For $u=(\log x) /(\log y) \geqslant 0$, we let $w(u)$ be the Buchstab's function (see [12, Sect. III.6]), and let $\rho(u)$ be the Dickman's function (see [12, Sect. III.5]). We let

$$
\mathcal{W}_{c}(x, y)= \begin{cases}\left(x w(c u)-y^{1 / c}\right) \frac{e^{\gamma}}{\zeta(1, y)}, & \text { in } H_{\varepsilon, c} \\ \frac{x}{\zeta(1, y)}, & \text { otherwise }\end{cases}
$$

where $\gamma$ is the Euler-Mascheroni constant and

$$
\zeta(1, y)=\prod_{\substack{p: \text { prime } \\ p \leqslant y}}\left(1-p^{-1}\right)^{-1}
$$

We put $H(u)=\exp \left(u / \log ^{2}(u+2)\right)$.

In our setting, we first derive an asymptotic for $\Phi_{c}(x, y)$ holding uniformly for a range of $y$ depending on $1<c<2229 / 1949=1.14366 \ldots$

Theorem 1 For $1<c<2229 / 1949$ fixed, there are positive numbers $\rho$ and $\eta$ such that for any $0<\varepsilon<\rho$ the following asymptotic formula holds

$$
\Phi_{c}(x, y)=\mathcal{W}(x, y)+O\left(\max \left\{x^{1-\eta}, R_{c}(x, y)\right\}\right)
$$

uniformly for

$$
2 \leqslant y< \begin{cases}\left\lfloor x^{c}\right\rfloor, & \text { when } 1<c<2509 / 2229 \\ x^{(1669-1389 c) / 280-\varepsilon}, & \text { when } 2509 / 2229 \leqslant c<2229 / 1949,\end{cases}
$$

where

$$
R_{c}(x, y)=\left\{\begin{array}{lc}
x \rho(c u)(\log y)^{-1}\left(H(c u)^{-a}+\exp \left(-(\log y)^{3 / 2-\varepsilon}\right)\right), & \text { in } H_{\varepsilon, c} \\
\log x\left(\max _{x^{c}} \geqslant t \geqslant y t^{\delta-1} \Psi(t, y)\right), & \text { otherwise. }
\end{array}\right.
$$

for some absolute constant $a>0$. 
Here we note that the error term in (1) is not sensitive to very small values of $y$. To illustrate this, given $1<c<2229 / 1949$, we take $y=\log ^{A}\left(x^{c}\right)$, for $A>c$. Then $t^{\delta-1} \Psi(t, y) \ll x^{\delta-1 / A+o(1)}$ (see [7]) uniformly for every $x^{c} \geqslant t \geqslant y$, and for large $x$.

$$
\Phi_{c}(x, y)-\frac{x}{\zeta(1, y)} \ll \max \left\{x^{1-\eta}, x^{1-\frac{c}{A}+o(1)}\right\} .
$$

Picking $A$ sufficiently close to $c$ and $x$ large, we see that $x^{1-\eta}$ dominates the other term in the maximum above.

We also remark that if $c \geqslant 2229 / 1949$ one may use sieve methods. It is not hard to show that

$$
\left\lfloor n^{c}\right\rfloor \equiv 0 \quad(\bmod d) \Longleftrightarrow\left\{\frac{n^{c}}{d}\right\}<\frac{1}{d} .
$$

Thus by Erdős-Turán inequality ([2, Theorem 2.1]), it follows that

$$
\#\left\{n \leqslant x:\left\lfloor n^{c}\right\rfloor \equiv 0 \quad(\bmod d)\right\}=\frac{x}{d}+E_{c}(x, d)
$$

where $E_{c}(x, d)$ satisfies

$$
\sum_{d \leqslant x^{\alpha}}\left|E_{c}(x, d)\right| \ll x^{1-\varepsilon}
$$

for some $\alpha=\alpha(c)$ by either van-der Corput's or Vinogradov's exponential sum estimates. Hence using Brun's Sieve we obtain

$$
\Phi_{c}(x, y) \asymp \frac{x}{\log y}
$$

uniformly for $2 \leqslant y \leqslant x^{\beta}$, where $\beta$ depends only on $c$. Furthermore with some work it can be shown that, using for instance Heath-Brown's recent $k$ th derivative test (see [4]), one has $\beta \sim a / c^{2}$ for some $a>0$ fixed. Since this result is rather weak compared to Theorem 1, we do not pursue this here.

Manuplating the Euler product of Dirichlet series of integers that are free of primes $\leqslant y$, one arrives at the convolution identity

$$
\sum_{\substack{d \mid n \\ P^{+}(d) \leqslant y}} \mu(d)= \begin{cases}1, & \text { if } P^{-}(n)>y, \\ 0, & \text { otherwise. }\end{cases}
$$

By Lemmas 1 and 2, the proof of the theorem reduces to exponential sums over rough numbers. Using the identity (3), one converts these exponential sums into exponential sums over integers that are free of large prime factors, in turn by Lemma 4, creating multinomial exponential sums of type (7). Treatment of these exponential sums differs depending on the size of $y$; that is, when $y$ is small, we use Lemma 9; when $y$ is large, we take into account the variation of the related parameters by combining Lemmas 9 and 10 . 


\subsection{Preliminaries and notation}

\subsubsection{Notation}

Given a real number $x$, we write $e(x)=e^{2 \pi i x},\{x\}$ for the fractional part of $x$ and $\lfloor x\rfloor$ for the greatest integer not exceeding $x$. The notation $\|x\|$ is used to denote the distance from the real number $x$ to the nearest integer. We write $n \sim N$ to mean that $n$ lies in a specified subinterval of $(N, 2 N]$. Furthermore, $c>1$ is a fixed real number and we put $\delta=1 / c$. Throughout of this paper $p$ always denotes a prime number. We recall that for functions $F$ and real nonnegative $G$ the notations $F \ll G$ and $F=O(G)$ are equivalent to the statement that the inequality $|F| \leqslant \alpha G$ holds for some constant $\alpha>0$. Further we use $F \asymp G$ to indicate that both $F \gg G$ and $F \ll G$ hold. We put

$$
\psi(x)=x-\lfloor x\rfloor-1 / 2, \text { and } \Delta \psi(x)=\psi\left(-(x+1)^{\delta}\right)-\psi\left(-x^{\delta}\right) .
$$

In a slight departure from convention, we shall frequently use $\varepsilon$ and $\eta$ to mean small positive numbers possibly not the same at each occurrence.

\subsubsection{Preliminaries}

In this section we state several lemmata that are to be used to reduce the proof of Theorem 1 to exponential sums.

Lemma 1 Let $c>1$. Suppose $\left\{a_{n}\right\}$ is a sequence of complex numbers with norm at most one. Then

$$
\sum_{n \leqslant x} a_{\left\lfloor n^{c}\right\rfloor}=\sum_{n \leqslant x^{c}} a_{n} \delta n^{\delta-1}+\sum_{n \leqslant x^{c}} a_{n} \Delta \psi(n)+O(1)
$$

where the implied constant depends only on $c$.

Proof The equality $m=\left\lfloor n^{c}\right\rfloor$ holds precisely when $m \leqslant n^{c}<m+1$, or equivalently, when $-(m+1)^{\delta} \leqslant n<-m^{\delta}$. Hence,

$$
\sum_{n \leqslant x} a_{\left\lfloor n^{c}\right\rfloor}=\sum_{m \leqslant x^{c}} a_{m}\left(\left\lfloor-m^{\delta}\right\rfloor-\left\lfloor-(m+1)^{\delta}\right\rfloor\right)+O(1) .
$$

The desired result follows upon recalling the facts

$$
(m+1)^{\delta}-m^{\delta}=\delta m^{\delta-1}+O\left(m^{\delta-2}\right) \quad(m \geqslant 1)
$$

and

$$
\sum_{m \leqslant x^{c}} a_{m} m^{\delta-2}=O(1)
$$

To deal with the penultimate term in Lemma 1, we use the following Lemma. 
Lemma 2 Suppose $1<y<z$. Let $H_{N} \geqslant 1$ be a real number. Then

$$
\begin{aligned}
\sum_{N<n \leqslant N^{\prime}} a_{n} \Delta \psi(n) \ll & N H_{N}^{-1}+H_{N}^{1 / 2} N^{\delta / 2}+H_{N}^{-1} \log H_{N} N^{1-\delta} \\
& +N^{\delta-1} \max _{N \leqslant N^{\prime \prime} \leqslant 2 N} \sum_{1 \leqslant h \leqslant H_{N}}\left|\sum_{N<n \leqslant N^{\prime \prime}} a_{n} e\left(h n^{\delta}\right)\right|
\end{aligned}
$$

for every $N<N^{\prime} \leqslant 2 N$, where the implied constant depends only on $\delta$.

Proof The proof follows using standard arguments, see e.g. [5, Sect. 4].

Lemma 3 For $H_{2} \geqslant H \geqslant H_{1} \geqslant 1$, let

$$
L(H)=\sum_{i=1}^{m} A_{i} H^{a_{i}}+\sum_{j=1}^{n} B_{j} H^{-b_{j}}
$$

where $A_{i}, B_{j}, a_{i}$ and $b_{j}$ are positive real numbers. Then

$$
\max _{H_{1} \leqslant H \leqslant H_{2}} L(H) \ll \sum_{i=1}^{m} A_{i} H_{1}^{a_{i}}+\sum_{j=1}^{n} B_{j} H_{2}^{-b_{j}}+\sum_{i=1}^{m} \sum_{j=1}^{n}\left(A_{i}^{b_{j}} B_{j}^{a_{i}}\right)^{1 /\left(a_{i}+b_{j}\right)}
$$

where the implied constants depends only on $m$ and $n$.

Proof See [5, Lemma 2.4.].

The following lemma allows one to factorize friable numbers in a convenient manner.

Lemma 4 Suppose that $2 \leqslant y \leqslant R \leqslant n \leqslant x$, with $P^{+}(n) \leqslant y$. Then there is a unique triple $(p, u, v)$ with,

(i) $n=p u v$

(ii) $p \leqslant y$

(iii) $R / p<v \leqslant R$ with $P^{-}(v) \geqslant p$ and $P^{+}(v) \leqslant y$

(iv) $u \leqslant x / p v$ with $P^{+}(u) \leqslant p$

Proof See e.g. [13, Lemma 10.1.].

Lemma 5 For any integer $\kappa \geqslant 3$, and every real number $\varepsilon>0$, there is an exponent pair given by

$$
(k, l)=\left(\frac{2}{(\kappa-1)^{2}(\kappa+2)}, 1-\frac{3 \kappa-2}{\kappa(\kappa-1)(\kappa-2)}+\varepsilon\right)
$$

Proof See [6, Theorem 2]. 


\subsubsection{Exponential sums with monomials}

For $H, K$ and $L$ positive integers, $X>0$ a real number; moreover $a(h, n)$ and $b(m)$ complex numbers with norm at most one, we define

$$
S=\sum_{H<h \leqslant 2 H} \sum_{K<n \leqslant 2 K} a(h, n) \sum_{L<m \leqslant 2 L} b(m) e\left(X \frac{h^{\beta} n^{\gamma} m^{\alpha}}{H^{\beta} K^{\gamma} L^{\alpha}}\right) .
$$

The following theorem is well-known result of Robert and Sargos (see e.g. [11]).

Lemma 6 Suppose $\alpha, \beta$ and $\gamma$ be real numbers such that $\alpha(\alpha-1) \beta \gamma \neq 0$. Then for every $\varepsilon>0$

$$
S \ll(H K L)^{1+\varepsilon}\left\{\left(\frac{X}{H K L^{2}}\right)^{1 / 4}+\frac{1}{(H K)^{1 / 4}}+\frac{1}{L^{1 / 2}}+\frac{1}{X^{1 / 2}}\right\} .
$$

where the implied constant depends only on $\alpha, \beta, \gamma$ and $\varepsilon>0$.

Lemma 7 Suppose $\alpha, \beta$ and $\gamma$ be real numbers such that $\alpha(\alpha-1) \beta \gamma \neq 0$. Further suppose $b(m)$ is an indicator function of a subinterval of $(L, 2 L]$. Then

$$
S \ll(H K L)^{1+\varepsilon}\left\{\left(\frac{X}{H K L^{2}}\right)^{1 / 4}+\frac{1}{L^{1 / 2}}+\frac{1}{X}\right\},
$$

where the implied constant depends only on $\alpha, \beta, \gamma$ and $\varepsilon>0$.

Proof See [11, Theorem 1].

The next result is a generalization of the method of estimating so called Type II sums in [6]. This was later generalized by Roger Baker (see e.g. [1]) to arbitrary exponent pairs under the assumption $X \geqslant H K$. Here we reprove his theorem in a slightly general manner.

Lemma 8 Let $(k, l)$ be an exponent pair. Suppose $\alpha<1$, and $\alpha \beta \gamma \neq 0$. Then

$$
\begin{aligned}
S \ll H L K \log (2 H K L)\{ & \left(\frac{X^{k}}{L^{1+k-l} H^{k} K^{k}}\right)^{1 / 2(k+1)}+\frac{1}{(H K)^{1 / 2}}+\frac{1}{X^{1 / 2}} \\
& \left.+\frac{1}{L^{(1+k-l) / 2(k+1)}}\right\}
\end{aligned}
$$

where the implied constant depends only on $\alpha, \beta, \gamma$ and $(k, l)$.

Proof We may assume $\frac{X^{k}}{L^{1+k-l} H^{k} K^{k}}<1$ and $X \geqslant 3$, otherwise the result is trivial. By Cauchy-Schwarz inequality one has

$$
S^{2} \ll L \sum_{L<m \leqslant 2 L}\left|\sum_{H<h \leqslant 2 H} \sum_{K<n \leqslant 2 K} a(h, n) e\left(X \frac{h^{\beta} n^{\gamma} m^{\alpha}}{H^{\beta} K^{\gamma} L^{\alpha}}\right)\right|^{2} .
$$


We now use [9, Lemma 5] with the choices $x_{i}=\frac{h_{1}^{\beta} n_{1}^{\gamma}}{2^{\beta+\gamma+1} H^{\beta} K^{\gamma}}$ and $z_{i}=X \frac{h^{\beta} n^{\gamma} m^{\alpha}}{H^{\beta} K^{\gamma} L^{\alpha}}$ yielding

$$
S^{2} \ll \frac{L}{\eta} \sum_{\substack{h_{1}, h_{2} \sim H \\ n_{1}, n_{2} \sim K \\|\Delta|<\eta}}\left|\sum_{m \sim L} e\left(2^{\gamma+\beta+1} X \Delta \frac{m^{\alpha}}{L^{\alpha}}\right)\right|
$$

where $\Delta=\frac{h_{1}^{\beta} n_{1}^{\gamma}-h_{2}^{\beta} n_{2}^{\gamma}}{2^{\beta+\gamma+1} H^{\beta} N^{\gamma}}$, and $1 / 2>\eta>0$ to be determined later. We next suppose $\eta \geqslant 1 / X$ and estimate the innermost sum trivially by $L$ whenever $|\Delta|<1 / X$, which by $[3$, Lemma 1$]$ gives rise to a contribution

$$
\ll \mathcal{E}:=\log (2 H K)\left\{\frac{L^{2} H K}{\eta}+\frac{H^{2} K^{2} L^{2}}{\eta X}\right\} .
$$

Applying the exponent pair $(k, l)$ whenever $\frac{1}{X} \leqslant|\Delta|<\eta$, it follows that

$$
S^{2} \ll \frac{L}{\eta} \sum_{\substack{h_{1}, h_{2} \sim H \\ n_{1}, n_{2} \sim K \\ \frac{1}{X} \leqslant|\Delta|<\eta}}\left\{X^{k}|\Delta|^{k} L^{l-k}+\frac{L}{X|\Delta|}\right\}+\mathcal{E} \ll \mathcal{I}_{1}+\mathcal{I}_{2}+\mathcal{E}
$$

where $\mathcal{I}_{1}$ and $\mathcal{I}_{2}$ denote the contribution of first and second terms in braces respectively. It is not too hard to see that splitting up the interval $\frac{1}{X} \leqslant|\Delta|<\eta$ into $\ll \log X \ll$ $\log (2 H K L)$ dyadic intervals and applying [3, Lemma 1] on each such interval, one has $\mathcal{I}_{2} \ll \log (2 H K L) \mathcal{E}$. Furthermore, by [3, Lemma 1], it follows that

$$
\mathcal{I}_{1} \log ^{-1}(2 H K) \ll \eta^{k-1} L^{1+l-k} X^{k} H K+\eta^{k} L^{1+l-k} X^{k} H^{2} K^{2} .
$$

Choosing $\frac{1}{X} \leqslant \eta<1 / 2$ optimally by Lemma 3, one gets

$$
\begin{array}{r}
S^{2} \log ^{-2}(2 H K L) \ll L^{2} H K+\frac{H^{2} K^{2} L^{2}}{X}+L^{1+l-k} X^{k} H K+L^{1+l-k} H^{2} K^{2} \\
+L^{\frac{1+l+k}{1+k}} H^{\frac{2+k}{1+k}} K^{\frac{2+k}{1+k}} X^{\frac{k}{1+k}}+H^{2} K^{2} L^{\frac{1+l+k}{1+k}}+L^{1+l-k} X^{k} H^{2-k} K^{2-k},
\end{array}
$$

hence the claimed result follows on using the assumptions that were made in the beginning.

The following result is useful when dealing with small $y$ 's in Theorem 1.

Lemma 9 Suppose $1<c<2229 / 1949$. Then for every exponent pair $(k, l)$ and every $\varepsilon>0$, there is a positive number $\eta=\eta(c, \varepsilon,(k, l))>0$ such that

$$
\sum_{\substack{1 \leqslant n \leqslant x^{c} \\ P^{-}(n)>y}} \Delta \psi(n) \ll \mathcal{I}_{c}(k, l, x, y) x^{\varepsilon}+x^{1-\eta},
$$


where $\mathcal{I}_{c}(k, l, x, y)$ is defined in (13) and the implied constant depends only on $c, \varepsilon$ and $(k, l)$.

Proof Let $0<\kappa</ 100$ be a small constant to be determined. Fixing $1<c<$ $2229 / 1949$, we may assume that $y \leqslant x^{\frac{c}{c+\kappa}}$, otherwise the result is trivial because of the factor $x^{1 / 2} y$ in $\mathcal{I}_{c}(k, l, x, y)$. Let $2 \leqslant y \leqslant M \leqslant x^{\frac{c}{c+\kappa}}$ be a parameter to be chosen. Then

$$
\sum_{\substack{1 \leqslant n \leqslant x^{c} \\ P^{-}(n)>y}} \Delta \psi(n)=\sum_{\substack{M^{c+\kappa}<n \leqslant x^{c} \\ P^{-}(n)>y}} \Delta \psi(n)+O\left(M^{c+\kappa}\right) .
$$

We next divide the interval $\left(M^{c+\kappa}, x^{c}\right]$ into intervals of the form $\left(N, N^{\prime}\right]$, where $N^{\prime}=\max \left\{2 N, x^{c}\right\}$. Let $0<\eta<1 / 1000$ be a constant to be chosen. Using Lemma 2 with $H_{N}=N^{1-\delta+\eta}$ on each such interval, we end up with estimating

$$
N^{\delta-1} \max _{N<N^{\prime} \leqslant 2 N} \sum_{1 \leqslant h \leqslant H_{N}} \varepsilon_{h} \sum_{\substack{N<n \leqslant N^{\prime} \\ P^{-}(n)>y}} e\left(h n^{\delta}\right)+N^{\delta-\eta}
$$

for some complex numbers $\varepsilon_{h}$ not exceeding 1 in modulus and for all $M^{c+\kappa}<N \leqslant x^{c}$.

To estimate the double sum above, we first use the convolution identity in (3) so as to convert it into

$$
S(N):=\sum_{1 \leqslant h \leqslant H_{N}} \varepsilon_{h} \sum_{\substack{d f \sim N \\ P^{+}(d) \leqslant y}} \mu(d) e\left(h(d f)^{\delta}\right) .
$$

On splitting ranges of $h, d, f$ into $\ll \log ^{3} N$ dyadic intervals one has

$$
S(N) \leqslant \sum_{\begin{array}{c}
H=2^{k} \leqslant H_{N} \\
K=2^{l} \leqslant N \\
L=2^{r} \leqslant N \\
K L \asymp N
\end{array}}|S(K, L, H)|
$$

where

$$
S(K, L, H)=\sum_{h \sim H} \varepsilon_{h} \sum_{\substack{d \sim K \\ f \sim L \\ d f \sim N \\ P^{+}(d) \leqslant y}} \mu(d) e\left(h(d f)^{\delta}\right) .
$$

Our first task is to show that, whenever $1<c<2229 / 1949$

$$
S(K, L, H) \ll N^{1-2 \eta+\varepsilon^{\prime}} \text { provided that } K \leqslant N^{\delta-5 \eta}
$$

uniformly for every $y$.

Let $\varepsilon^{\prime}>0$ be a small number. A straightforward application of Lemma 7 yields

$$
S(K, L, H) \ll N^{1-2 \eta+\varepsilon^{\prime}} \text { provided that } K \leqslant N^{3 \delta-2-12 \eta}
$$


uniformly for all $y$. As for the case $N^{\delta-5 \eta} \geqslant K>N^{3 \delta-2-12 \eta}$ : using the elementary estimate

$$
\int_{-1 / 2}^{1 / 2}\left|\sum_{m \in I_{N}} e(\alpha m)\right| d \alpha \ll \int_{-1 / 2}^{1 / 2} \min \left\{\frac{1}{\|\alpha\|}, N\right\} d \alpha \ll \log 2 N \quad(N \geqslant 1)
$$

holding for any sub-interval of $I_{N} \subset[N, 2 N)$, it follows that for some complex numbers $a(h, f)$ and $b(d)$ not exceeding 1 in modulus, one has

$$
\begin{aligned}
S(K, L, H)= & \int_{-1 / 2}^{1 / 2} \sum_{h \sim H} \sum_{f \sim L} a(h, f) \sum_{d \sim K} b(d) e(-\alpha d) e\left(h(d f)^{\delta}\right) \sum_{m \sim N / f} e(\alpha m) d \alpha \\
& \ll \log N \sup _{\alpha \in[-1 / 2,1 / 2]}\left|\sum_{h \sim H} \sum_{f \sim L} a(h, f) \sum_{d \sim K} b(d) e(-\alpha d) e\left(h(d f)^{\delta}\right)\right| .
\end{aligned}
$$

Lemma 8 yields

$$
\begin{aligned}
& S(K, L, H) \log ^{-2} N \ll\left(H_{N}^{1 / 2} N^{1-\delta / 2}+H_{N}^{1 / 2} N^{1 / 2} K^{1 / 2}\right. \\
& \left.\quad+H_{N} N K^{-(1+k-l) /(2(k+1))}+H_{N} N^{(2+k(\delta+1)) /(2(k+1))} K^{-(1-l) / 2((1+k))}\right),
\end{aligned}
$$

where $(k, l)$ is an exponent pair. Examining the worst scenario, we derive that

$$
S(K, L, H) \ll N^{1-2 \eta} \log ^{2} N
$$

holds whenever

$$
N^{(1-\delta)(k+2) /(1-l)+6 \eta(k+1) /(1-l)} \leqslant K \leqslant N^{\delta-5 \eta} .
$$

In order that this last estimate is applicable whenever $K>N^{3 \delta-2-12 \eta}$, we should have

$$
3 \delta-2-12 \eta>(1-\delta)(k+2) /(1-l)+6 \eta(k+1) /(1-l) .
$$

This is satisfied if $1<c<\frac{k-3 l+5}{k-2 l+4}$ and that $\eta$ sufficiently small. To get $1<c<$ $2229 / 1949$, we pick $(k, l)=B(1 / 162,359 / 378+\varepsilon)=(85 / 189+\varepsilon, 41 / 81)$. Here the exponent pair $(1 / 162,359 / 378+\varepsilon)$ is obtained by taking $\kappa=7$ in Lemma 5 .

To handle the case $K>N^{\delta-5 \eta}$, we choose $\kappa=5 \eta c /(\delta-5 \eta)$ so that the inequality $(\delta-5 \eta)(c+\kappa)=1$ is satisfied, yielding

$$
y \leqslant M \leqslant M^{(c+k)(\delta-5 \eta)} \leqslant N^{\delta-5 \eta} \leqslant K<d \leqslant 2 K .
$$


It hence follows by Lemma 4 that

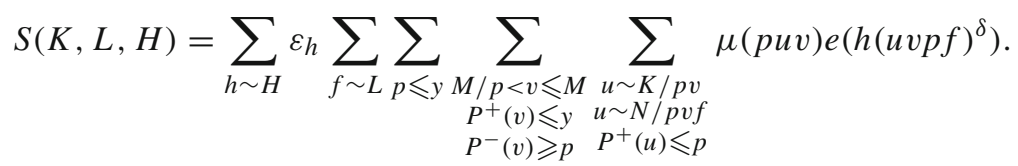

Here it is clear that the presence of the factor $\mu(p u v)$ makes the inequalities $P^{+}(u) \leqslant$ $p$ and $P^{-}(v) \geqslant p$ strict, allowing one to split the möbius function:

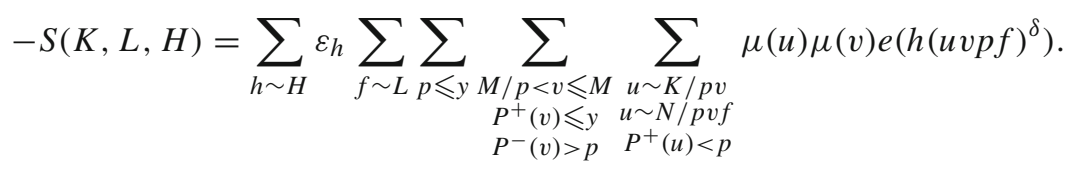

On splitting the ranges of $p, v, u$ into dyadic intervals, we are to estimate

$$
S(K, L, H, U, V, P):=\sum_{\substack{h \sim H \\ f \sim L \\ u \sim U}} \varepsilon_{h} \mu(u) \sum_{\substack{p \sim P \\ v \sim V \\ P^{+}(u)<p \\ u v f p \sim N \\ u v p \sim K}} a(v, p) e\left(h(\text { uvpf })^{\delta}\right) .
$$

for some $a(v, p)$ complex numbers not exceeding one in modulus. We next note that $S(K, L, H, U, V, P)$ is

$$
\begin{aligned}
\int_{-1 / 2}^{1 / 2} \int_{-1 / 2}^{1 / 2} \sum_{\substack{h \sim H \\
f \sim L \\
u \sim U}} \varepsilon_{h} \mu(u) & \sum_{\substack{p \sim P \\
v \sim V}} a(v, p) e\left(h(u v p f)^{\delta}+\alpha p v+\beta p\right) \\
& \sum_{\substack{n f u \sim N \\
n u \sim K}} e(-\alpha n) \sum_{\substack{m \sim P \\
P^{+}(u)<m}} e(-m \beta) d \alpha d \beta
\end{aligned}
$$

for some $a(v, p)$ complex numbers not exceeding one in modulus. Here the last expression, by (9), equals

$$
\ll \log ^{2} N \sum_{\substack{h \sim H \\ f \sim L \\ u \sim U}}\left|\sum_{\substack{p \sim P \\ v \sim V}} a^{\prime}(v, p) e\left(h(u v p f)^{\delta}\right)\right|
$$

for some $\left|a^{\prime}(v, p)\right| \leqslant 1$. Grouping the terms $r=u f$ and $r^{\prime}=p v$, it follows that $S(K, L, H, U, V, P)$ is

$$
\ll \sum_{\substack{h \sim H \\ r \asymp L U}} c(r)\left|\sum_{r^{\prime} \asymp P V} c^{\prime}\left(r^{\prime}\right) e\left(h\left(r r^{\prime}\right)^{\delta}\right)\right|
$$


for some $|c(r)|,\left|c^{\prime}\left(r^{\prime}\right)\right| \leqslant d(r)$, where $d(r)$ is the divisor function. Using the bound $d(r) \ll r^{\varepsilon}$ and the relations $L U P V \asymp N, M / P \ll V \ll M$, Lemma 8 yields

$$
\begin{array}{r}
S(K, L, H, U, V, P) N^{-2 \varepsilon} \ll H N^{\frac{k(\delta+1)+2}{2(k+1)}} M^{-\frac{1-l}{2(k+1)}}+H^{1 / 2} N^{1 / 2} P^{1 / 2} M^{1 / 2} \\
H N M^{-\frac{1+k-l}{2(k+1)}}+H^{1 / 2} N^{1-\delta / 2},
\end{array}
$$

thus

$$
\begin{array}{r}
S(K, L, H) N^{-3 \varepsilon} \ll H N^{\frac{k(\delta+1)+2}{2(k+1)}} M^{-\frac{1-l}{2(k+1)}}+H^{1 / 2} N^{1 / 2} P^{1 / 2} M^{1 / 2} \\
H N M^{-\frac{1+k-l}{2(k+1)}}+H^{1 / 2} N^{1-\delta / 2}
\end{array}
$$

whenever $K \geqslant N^{\delta-5 \eta}$.

Summing over $K, L$ and $H$ subject to the bounds in (8), (11), (12) and plugging the resulting estimate into (6), finally summing over $N$, we get

$$
\begin{array}{r}
\sum_{\substack{1 \leqslant n \leqslant x^{c} \\
P^{-}(n)>y}} \Delta \psi(n) \ll x^{3 \varepsilon+\eta}\left(x^{\frac{k(c+1)+2 c}{2(k+1)}} M^{-\frac{1-l}{2(k+1)}}+x^{1 / 2} y^{1 / 2} M^{1 / 2}\right. \\
\left.+x^{c} M^{-\frac{1+k-l}{2(k+1)}}+M^{c}\right)+x^{1-\eta}
\end{array}
$$

Choosing $y \leqslant M \leqslant x^{\frac{c}{c+\kappa}}$ optimally by Lemma 3 we get

$$
\begin{array}{ll}
\sum_{\substack{1 \leqslant n \leqslant x^{c} \\
P^{-}(n)>y}} \Delta \psi(n) & \ll x^{4 \varepsilon}\left(x^{\frac{k(c+1)+2 c+1-l}{2(k+1)}}+x^{1 / 2} y+x^{c-\frac{(1+k-l)}{2(k+1)}}+y^{c}+x^{\frac{2 c^{2}(k+1)}{2 c(k+1)+1+k-l}}\right. \\
& \left.x^{\frac{c(k(c+1)+2 c)}{2(k+1) c+l-1}}+x^{\frac{c(k+2)+k+1-l}{2(2+k-l)}} y^{\frac{1-l}{2(2+k-l)}}+x^{\frac{1+k-l+2 c(k+1)}{2(2+2 k-l)}} y^{\frac{1+k-l}{2(2+2 k-l)}}\right) \\
& +x^{1-\eta} \\
& :=x^{4 \varepsilon} \mathcal{I}_{c}(k, l, x, y)+x^{1-\eta} .
\end{array}
$$

Replacing $4 \varepsilon$ by $\varepsilon$ the desired result follows.

If $y \geqslant x^{4 / 5}$ (say), Lemma 9 provides no usefull information. Hence, for large values of $y$ we use the following lemma.

Lemma 10 Suppose $1<c<2$. Then for every $\varepsilon>0$, there is a positive real number $\eta=\eta(\varepsilon, c)$ such that

$$
\sum_{\substack{1 \leqslant n \leqslant x^{c} \\ L<P^{-}(n) \leqslant y}} \Delta \psi(n) \ll x^{\varepsilon}\left\{x^{\frac{3 c+1}{4}} L^{-1 / 4}+x^{\frac{2 c+1}{4}} y^{1 / 4}+x^{c} L^{-1 / 2}\right\}+x^{1-\eta},
$$

uniformly for every $1 \leqslant L<y \leqslant x^{c}$. 
Proof We split the sum into $\ll \log x$ dyadic sums of the form $\left(N, N^{\prime}\right]$, where $N^{\prime}=$ $\max \left(2 N, x^{c}\right)$, then apply Lemma 2 with $H_{N}=N^{1-\delta+\eta}$. Thus for $N \geqslant 2$ we are to estimate

$$
\begin{aligned}
S(N) & =\sum_{1 \leqslant h \leqslant H_{N}}\left|\sum_{\substack{N<n \leqslant N^{\prime} \\
L<P^{-}(n) \leqslant y}} e\left(h n^{\delta}\right)\right|=\sum_{1 \leqslant h \leqslant H_{N}}\left|\sum_{\substack{L<p \leqslant y \\
N / p<n \leqslant N^{\prime} / p \\
P^{-}(n) \geqslant p}} e\left(h p^{\delta} n^{\delta}\right)\right| \\
& =\sum_{1 \leqslant h \leqslant H_{N}} \varepsilon_{h} \sum_{\substack{L<p \leqslant R \\
L}} \sum_{\substack{N<n \leqslant N^{\prime} / p \\
P^{-}(n) \geqslant p}} e\left(h p^{\delta} n^{\delta}\right)
\end{aligned}
$$

for some $\varepsilon_{h}$ complex number with modulus at most 1 . We next divide all the ranges of $h, p$ and $n$ into dyadic intervals:

$$
S(N)=\sum_{\substack{H \leqslant H_{N} \\ H=2^{k}}} \sum_{\substack{L<P \leqslant y \\ P=2^{s}}} \sum_{\substack{N<X \leqslant 2 N^{\prime} / P \\ X=2^{m}}} S(X, P, H)
$$

where

$$
S=S(X, P, H)=\sum_{h \sim H} \sum_{n \sim X} \sum_{\substack{p \sim P \\ n p \sim N \\ P^{-}(n) \geqslant p}} \varepsilon_{h} e\left(h n^{\delta} p^{\delta}\right) .
$$

The conditions $P^{-}(n) \geqslant p$ and $n p \sim N$ may be omitted as before, yielding

$$
S \ll \log P \sup _{\alpha \in[-1 / 2,1 / 2]}\left|\sum_{h \sim H} \sum_{n \sim X} \sum_{p \sim P} a(n, h) e(\alpha p) e\left(h n^{\delta} p^{\delta}\right)\right|
$$

for some complex number $a(n, h)$ with modulus at most 1 . Applying Lemma 6 together with the relation $P X \asymp N$ and summing over $H, X$ and $P$ yields

$$
\begin{array}{r}
S(N) N^{\delta-1-\varepsilon / 2} \ll N^{\delta-1}( \\
H_{N} N^{\frac{3+\delta}{4}} L^{-1 / 4}+H_{N}^{3 / 4} N^{3 / 4} y^{1 / 4} \\
\left.+H_{N} N L^{-1 / 2}+H_{N}^{1 / 2} N^{1-\delta / 2}\right) .
\end{array}
$$

The desired result now follows by summing over $N$.

\subsection{Proof of Theorem 1}

Proof Lemma 1 implies

$$
\Phi_{c}(x, y)=\sum_{\substack{n \leqslant n^{c} \\ P^{-}(n)>y}} \delta n^{\delta-1}+\sum_{\substack{n \leqslant x^{c} \\ P^{-}(n)>y}} \Delta \psi(n)+O(1) .
$$


We first obtain the main term. Combining [12, Theorem 7] and [12, Entry 51] together with the partial summation formula, one obtains

$$
\sum_{\substack{n \leqslant x^{c} \\ P^{-}(n)>y}} \delta n^{\delta-1}=\frac{x}{\zeta(1, y)}+O\left(\log x\left(\max _{x^{c} \geqslant t \geqslant y} t^{\delta-1} \Psi(t, y)\right)\right)
$$

hence the desired result when $(x, y) \notin H_{\varepsilon, c}$.

For the case $(x, y) \in H_{\varepsilon, c}$, we first record the following result.

Lemma 11 Suppose $a \geqslant 0$, and let $1<c<2$ be a fixed number. For every $(x, y) \in$ $H_{c, \varepsilon}$ the following estimate holds

$$
\int_{y}^{x^{c}} \frac{t^{\delta-1}}{H^{a}\left(\frac{\log t}{\log y}\right)} \rho\left(\frac{\log t}{\log y}\right) d t \ll \frac{x \rho(c u)}{H^{a}(c u)} .
$$

Proof We may assume that $y \leqslant x^{c / 3}$, otherwise, since $\rho(v)$ and $H(v)$ are uniformly bounded, the result is trivial.

Let $f(t)=\frac{t^{\delta-1 / 2}}{H^{a}\left(\frac{\log t}{\log y}\right)} \rho\left(\frac{\log t}{\log y}\right)$ be defined for all $x^{c} \geqslant t \geqslant y$. It is suffices to show that the relation

$$
f(t) \leqslant C f\left(x^{c}\right)
$$

holds for some constant $C$ independent of $y$. Assume $x^{c} \geqslant t \geqslant y^{3}$. Using [12, Lemma $8.1]$ and [12, Corollary 8.3], it follows that

$f^{\prime}(t)=t^{\delta-3 / 2} \rho(v) H^{-a}(v)\left\{\delta-1 / 2-\frac{\log (v \log v)}{\log y}\left(1+O\left(\frac{\log \log v}{\log ^{2} v}\right)\right)-a \frac{H^{\prime}(v)}{H(v) \log y}\right\}$

where $v=\log t / \log y \geqslant 3$. Thus for any fixed $1<c<2$, given $\varepsilon>0$ and $x$ large $f^{\prime}(t)>0$ is satisfied when $(x, y) \in H_{\varepsilon, c}$. This proves (14) uniformly in $y$, whenever $x^{c} \geqslant t \geqslant y^{3}$. As for the case: $x^{c} \geqslant y^{3} \geqslant t \geqslant y$, (14) is satisfied uniformly, since $\rho(v)$ and $H(v)$ are bounded, we have

$$
f(t) \leqslant C^{\prime} f\left(y^{3}\right) \leqslant C C^{\prime} f\left(x^{c}\right)
$$

for some constant $C^{\prime}$, hence the result.

Riemann-Stieltjes integration together with [12, Corollary 7.5] implies that

$$
\begin{aligned}
\sum_{\substack{n \leqslant n^{c} \\
P^{-}(n)>y}} \delta n^{\delta-1}= & \frac{1}{\zeta(1, y)} \int_{y}^{x^{c}} \delta t^{\delta-1} w\left(\frac{\log t}{\log y}\right) d t \\
& +\frac{1}{\zeta(1, y) \log y} \int_{y}^{x^{c}} \delta t^{\delta-1} w^{\prime}\left(\frac{\log t}{\log y}\right) d t+\int_{y^{-}}^{x^{c}} \delta t^{\delta-1} d[R(t, y)]
\end{aligned}
$$


where

$$
R(t, y)=\frac{x \rho(\log t / \log y)}{\log ^{2} y}\left\{H^{-a}(\log t / \log y)+\exp \left\{(\log y)^{-3 / 2+\varepsilon}\right\}\right\}
$$

Noting the upper bound $w^{\prime}(u) \ll \rho(u) H^{-a^{\prime}}(u)$ for some $a^{\prime}>0$ and for every $u \geqslant 0$ (see [12, Theorem 6])), partial summation and Merten's theorem (see [12, Theorem 11]) yield

$$
\begin{array}{r}
\frac{1}{\zeta(1, y)} \int_{y}^{x^{c}} \delta t^{\delta-1} w\left(\frac{\log t}{\log y}\right) d t=\left(x w(c u)-y^{\delta}\right) \frac{e^{\gamma}}{\zeta(1, y)} \\
+O\left(\frac{1}{\log ^{2} y} \int_{y}^{x^{c}} \frac{t^{\delta-1}}{H^{a}\left(\frac{\log t}{\log y}\right)} \rho\left(\frac{\log t}{\log y}\right) d t\right) .
\end{array}
$$

Lemma 11 now implies that,

$$
\sum_{\substack{n \leqslant n^{c} \\ P^{-}(n)>y}} \delta n^{\delta-1}-\left(x w(c u)-y^{\delta}\right) \frac{e^{\gamma}}{\zeta(1, y)} \ll \frac{x \rho(c u)}{\log ^{2} y}\left\{H^{-a}(c u)+\exp \left\{(\log y)^{-3 / 2+\varepsilon}\right\}\right\} .
$$

Having derived the main term, we are now required to show that

$$
\sum_{\substack{n \leqslant x^{c} \\ P^{-}(n)>y}} \Delta \psi(n) \ll x^{1-\eta}
$$

holds uniformly for aforementioned ranges of $y$ depending on $c$. We shall do this in two steps. In the first step, we use Lemma 9 with $(k, l)=(85 / 189+\varepsilon, 41 / 81)$ giving

$$
\begin{aligned}
& \sum_{\substack{n \leqslant x^{c} \\
P^{-}(n)>y}} \Delta \psi(n) \ll x^{\varepsilon}\left(y^{c}+x^{1 / 2} y+x^{\frac{1389 c+535}{2204}} y^{\frac{70}{551}}+x^{\frac{1644 c+535}{2714}} y^{\frac{535}{2714}}\right)+x^{1-\eta} \\
& \\
& \quad+x^{\varepsilon}\left(x^{\frac{1389 c^{2}+255 c}{1644 c+280}}+x^{\frac{1644 c^{2}}{1644 c+535}}+x^{\frac{926 c-25}{1644}}+x^{\frac{1644 c-535}{1644}}\right)
\end{aligned}
$$

uniformly for every $y \leqslant x^{c}$. Here, the last four terms may be eliminated at the cost of assuming $1<c<1.1719$.

If $2509 / 2229 \leqslant c<2229 / 1949$, only the third term survives, thereby proving Theorem 1 for the second range in (2).

To prove Theorem 1 for the first range in (2), we may assume that $y>$ $\left\lfloor x^{3(c-1)+10 \eta}\right\rfloor$, otherwise it is easy to see that the left hand side of (16) is $x^{1-\eta}$ whenever $y \leqslant\left\lfloor x^{3(c-1)+10 \eta}\right\rfloor$. 
We note that

$$
\begin{aligned}
\sum_{\substack{n \leqslant x^{c} \\
P^{-}(n)>y}} \Delta \psi(n) & =\sum_{\substack{n \leqslant x^{c} \\
P^{-}(n) \geqslant L}} \Delta \psi(n)-\sum_{\substack{n \leqslant x^{c} \\
y \geqslant P^{-}(n)>L}} \Delta \psi(n) \\
& =\sum_{\substack{n \leqslant x^{c} \\
P^{-}(n)>L}} \Delta \psi(n)-\sum_{\substack{n \leqslant x^{c} \\
y \geqslant P^{-}(n)>L}} \Delta \psi(n)+O\left(x^{c} / L\right)
\end{aligned}
$$

where the error is due to the possible sum $\sum_{\substack{n \leqslant x^{c} \\ P^{-}(n)=L}} \ll x^{c} / L$.

We may choose $L=\left\lfloor x^{3(c-1)+10 \eta}\right\rfloor$ making the first sum $\ll x^{1-\eta}$. With this choice of $L$, by Lemma 10 , the second sum and the third term above is $\ll x^{1-\eta}$, provided that $y \leqslant x^{3-2 c-\varepsilon}$ and that $1<c<2509 / 2229$.

To get the range $y \leqslant\left\lfloor x^{c}\right\rfloor$, we note that for $x^{c}>y \geqslant x^{c / 2}$ one has

$$
\sum_{\substack{n \leqslant x^{c} \\ P^{-}(n)>y}} \Delta \psi(n)=O(1)+\sum_{p \leqslant x^{c}} \Delta \psi(p)-\sum_{p \leqslant y} \Delta \psi(p) \ll x^{1-\eta},
$$

by [9, Theorem 1] whenever $1<c<2817 / 2426$. Since $3-2 c>c / 2$, the desired result follows.

\section{References}

1. Baker, R.C.: The square-free divisor problem. Q. J. Math. Oxf. Ser. (2) 45(179), 269-277 (1994)

2. Baker, R.C.: Diophantine inequalities. Lond. Math. Soc. Monogr. (N.S.) 1 (Clarendon Press, New York) (1986)

3. Fouvry, E., Iwaniec, H.: Exponential sums with monomials. J. Number Theory 33(3), 311-333 (1989)

4. Heath-Brown, D.R.: A New $k$-th Derivative Estimate for Exponential Sums via Vinogradov's Mean Value. arXiv:1601.04493

5. Graham, S.W., Kolesnik, G.: van der Corput's method of exponential sums. In: London Mathematical Society Lecture Note Series, vol. 126. Cambridge University Press, Cambridge (1991) (ISBN 0-52133927-8)

6. Heath-Brown, D.R.: The Pjatecki-Sapiro prime number theorem. J. Number Theory 16(2), 242-266 (1983)

7. Hildebrand, A.: On the number of positive integers $\leq x$ and free of prime factors $y$. J. Number Theory 22, 289-307 (1986)

8. Piatetski-Shapiro, I.I.: On the distribution of prime numbers in sequences of the form $\lfloor f(n)\rfloor$ (Russian). Mat. Sb. N.S. 33 (75), 559-566 (1953)

9. Rivat, J., Sargos, P.: Nombres premiers de la forme $\left\lfloor n^{c}\right\rfloor$. Can. J. Math 53(2), 414-433 (2001)

10. Rivat, J., Wu, J.: Prime numbers of the form $\left\lfloor n^{c}\right\rfloor$. Glasg. Math. J. 43(2), 237-254 (2001)

11. Robert, O., Sargos, P.: Three-dimensional exponential sums with monomials. J. Reine Angew. Math. 591, 1-20 (2006)

12. Tenenbaum, G.: Introduction to analytic and probabilistic number theory. In: Cambridge Studies in Advanced Mathematics, vol. 46. Cambridge University Press, Cambridge (1995) (ISBN: 0-521-412617)

13. Vaughan, R.C.: A new iterative method in Waring's problem. Acta Math. 162(1-2), 1-71 (1989) 